\title{
Intracranial venous thrombosis in the first trimester of pregnancy
}

\author{
P. J . M . L A V I N, I. B O N E, J . T. L A M B, A N D L. M . S W I N B U R E \\ From the Departments of Neurology, Pathology, and Neuroradiology, St James's University Hospital \\ and Chapel Allerton Hospital, Leeds
}

SUMMARY We describe a fatal case of intracranial venous thrombosis occurring in early pregnancy. Such thrombosis usually occurs in late pregnancy or the puerperium but rarely during the first trimester of pregnancy. Computerised axial tomography suggested massive cerebral venous infarction. Necropsy findings showed not only cerebral venous thrombosis but also extensive pelvic and iliac vein thromboses. The relationship of cerebral venous thrombosis and pregnancy is discussed and the literature reviewed.

Cerebral venous and dural sinus thrombosis are known to occur in a variety of conditions including trauma, infection (particularly paranasal sinuses and middle ear), severe anaemia, hypercoagulable states, dehydration, and debilitating diseases (Kalbag and Woolf, 1967; Gettelfinger and Kokmen, 1977).

Gowers (1888) suggested an association of cerebral venous thrombosis, among other things, with pregnancy. In late pregnancy and in the puerperium such an association is now well recognised, having an incidence of one in 3000 pregnancies (Huggenberg and Kesselring, 1958). Often it is found to occur in pregnancy with obstetric complications (Garcin and Pestel, 1955), but it is exceedingly rare in the first trimester of pregnancy, there being only a few pathologically proven cases described (Meinel, 1901; Fishman et al., 1957; Hensell, 1961; Eckerling et al., 1963; Kalbag and Woolf, 1967; Christensen et al., 1969). Diagnosis usually depends on angiography. Indeed Kalbag and Woolf (1967) state that "without it intra vitam diagnosis can never be more than a guess." The case we report underwent computerised axial tomography which suggested massive cerebral venous infarction of both hemispheres with haemorrhage and supported the clinical diagnosis of intracranial venous thrombosis.

Address for reprint requests: Dr P. Lavin, Department of Neurology, Charing Cross Hospital, Fulham Palace Road, London W6 8RF.

Accepted 22 February 1978

\section{Case report}

A 42 year old Asian housewife was admitted to an obstetric unit after a generalised tonic/clonic $\triangle$ seizure. She was eight weeks pregnant, and had complained of frontal headaches, vomiting, and insomnia for three weeks before admission. Her relatives gave a history of possible seizures for several years. She had had five uneventful pregnancies.

Initial examination showed her to be apyrexial, drowsy with no papilloedema or focal neurological signs. She was normotensive. She had five further seizures despite intravenous anticonvulsant therapy. Between seizures it was noted that she was becoming less responsive, and on the fourth day she was transferred to the Neurological Department.

On arrival she was in an akinetic state, responding only to noxious stimuli. There was a paucity of movement on the left side with an extensor plantar response. The fundi were normal. No further seizures occurred.

Investigations showed an ESR of $14 \mathrm{~mm} / \mathrm{hr}$; haemoglobin $15.5 \mathrm{gm} / \mathrm{dl}$ with a normal blood film; plasma urea and electrolytes normal; random blood sugar $7.0 \mathrm{mmol} / \mathrm{l}$. The skull and chest radiographs were normal. The EEG showed diffuse slow waves alternating with low voltage rapid activity. A lumbar puncture demonstrated clear cerebrospinal fluid with a pressure of $300 \mathrm{~mm} \mathrm{CSF}$; white cells $3 \times 10^{3} / \mathrm{ml}$; protein $0.23 \mathrm{gm} / 1$; no organisms were seen and the culture was negative. 
Computerised axial tomography before and after intravenous contrast medium $(50 \mathrm{ml}$ of sodium iothalamate, $420 \mathrm{mg}$ iodine $/ \mathrm{ml}$ ) showed massively decreased absorption deeply throughout both cerebral hemispheres, more marked on the right, with some peripheral sparing. There was partial involvement of the basal ganglia. The lateral ventricles were small in size but normal in position (Fig. 1). Two small, high density areas were seen towards the vertex (Fig. 2) suggestive of haemorrhage. The appearances of the CT scan suggested massive cerebral venous infarction with oedema.

The patient continued to deteriorate and died on her sixth day in hospital.

\section{Necropsy findings}

\section{BRAIN AND MENINGES}

The dura mater was tense and the brain evenly swollen with flattened convolutions. There were areas of haemorrhagic infarction in the medial portions of both parietal lobes which extended to the surface over the vertex, but spared the grey matter on either side of the central sulcus (Fig. 3). That on the right was more extensive, impinging on the medial surface and extending anteriorly

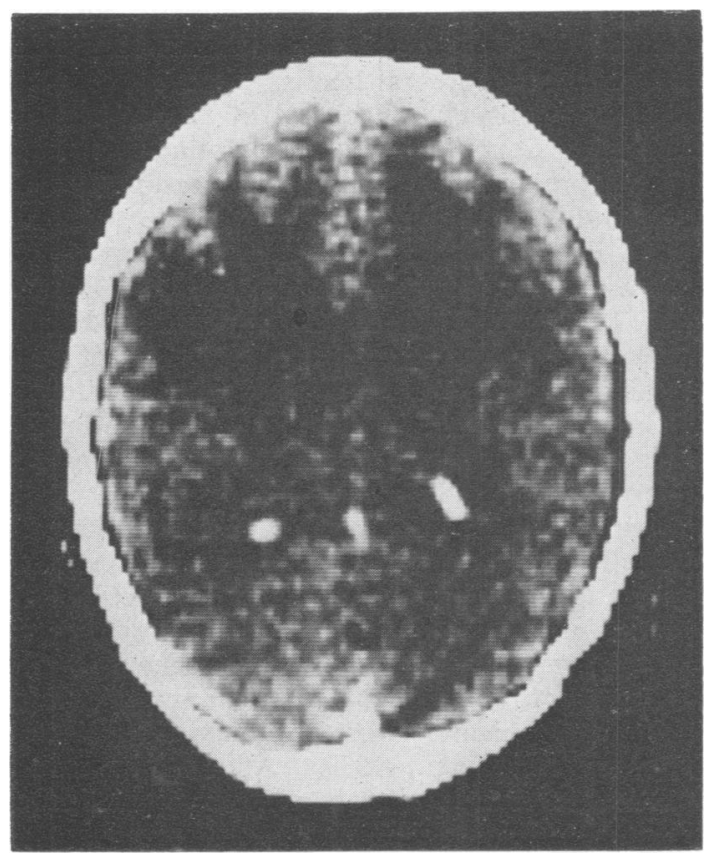

Fig. 1 CT scan showing massively decreased absorption in both cerebral hemispheres, more marked on the right, with small lateral ventricles.

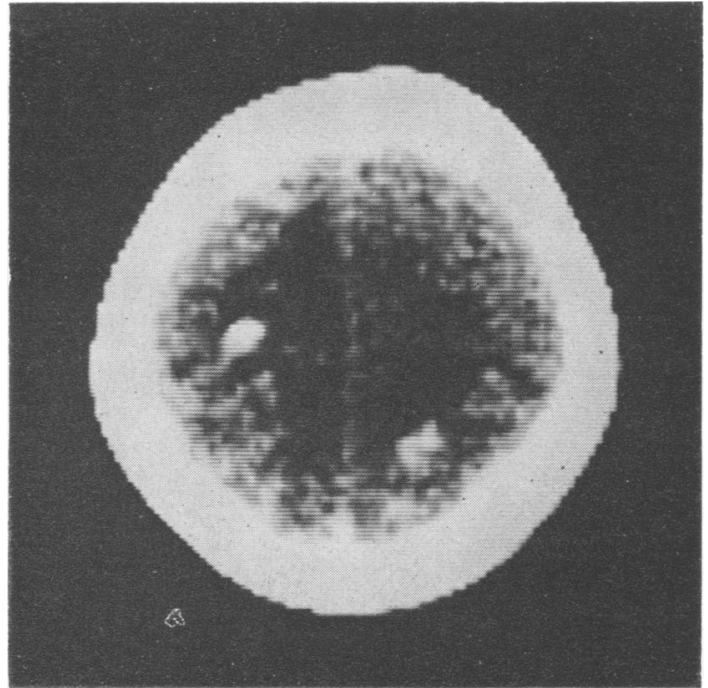

Fig. $2 C T$ scan showing two high density areas towards the vertex, suggestive of haemorrhage.

and posteriorly to the anterior end of the occipital lobe (Fig. 4). On the left side there was a crescentic area of haemorrhage $40 \times 10 \mathrm{~mm}$ on the deep surface of the infarct. Petechial haemorrhages in the softened cerebral tissue surrounded the main areas of dense infarction. The dural veins and both lateral and sigmoid sinuses contained soft antemortem thrombus. The smaller veins contained clot of the agonal type. There was slight, cerebellar coning with trivial tentorial notching. The internal carotid arteries, the circle of Willis, and the cavernous sinuses were normal.

\section{OTHER FINDINGS}

The pelvic veins contained antemortem thrombus which extended to the common iliac veins, mainly down the left side, even to the femoral and the posterior tibial veins, and which was firmly adherent in places.

There were one or two thrombi in the peripheral branches of the right main pulmonary artery but no infarction.

The uterus contained an $18 \mathrm{~mm}$ embryo with no visible abnormality. The placenta was partially separated.

There was a large nodular goitre.

\section{Discussion}

Cerebral venous thrombosis is a well-recognised complication of late pregnancy or the puerperium (Kalbag and Woolf, 1967) and has been the subject of an extensive review (Carroll et al., 1966). It 


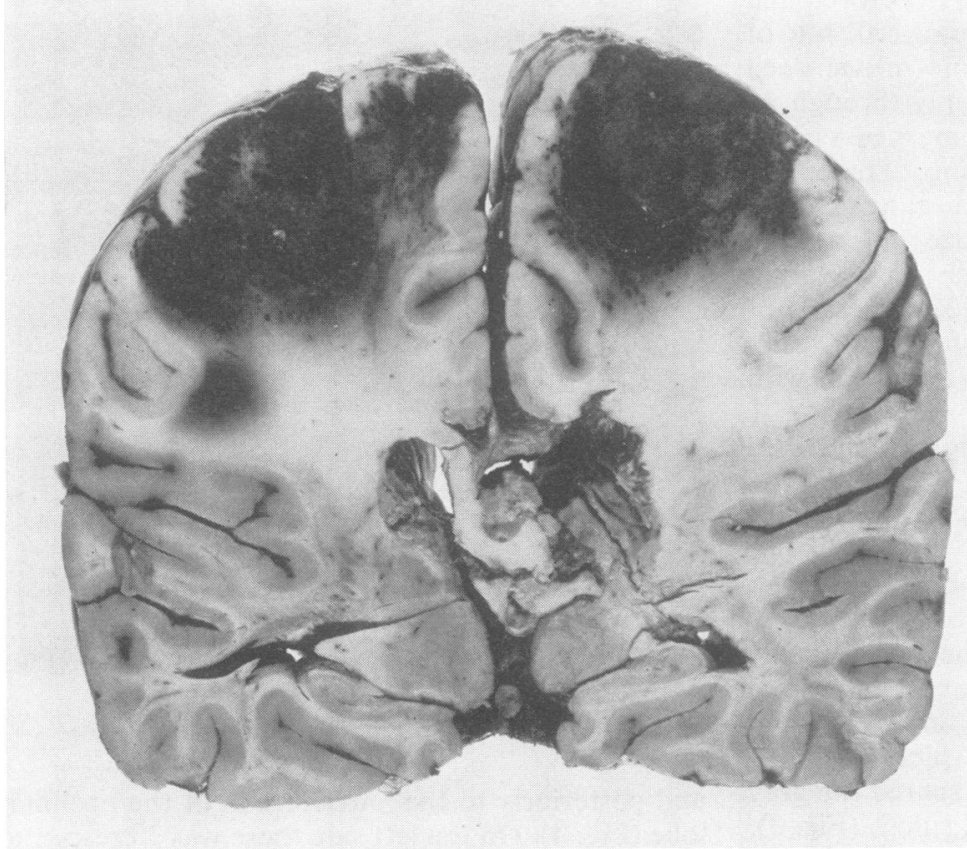

Fig. 3 Coronal section of brain showing extensive areas of haemorrhagic infarction in both cerebral hemispheres.

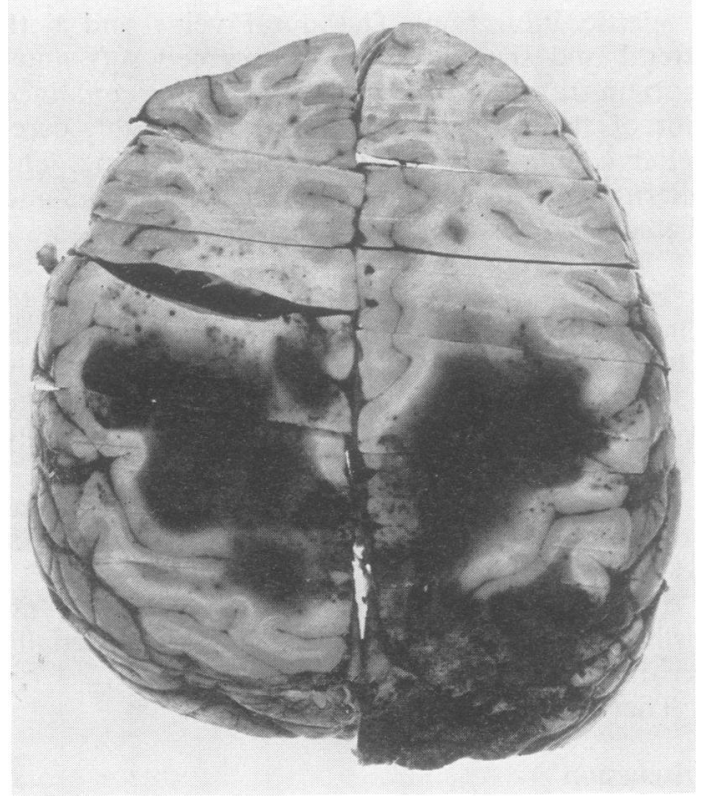

Fig. 4 Brain sectioned in horizontal plane, showing haemorrhagic infarction of both cerebral hemispheres, more marked on the right. may take the form of superior sagittal sinus thrombosis or be more advanced, with involvement of the cortical veins and haemorrhagic infarction of the cerebral hemispheres. The clinical picture depends on the extent of thrombosis. In pregnancy, Carroll et al. (1966) found that 34\% of cases presented with headache, presumed to be due to raised intracranial pressure, while $30 \%$ presented with seizures. The prognosis is variable with a mortality rate of between $33 \%$ (Fishman et al., 1957) and 56\% (Huggenberg and Kesselring, 1958). Treatment is symptomatic; the role of anticoagulants and fibrinolytic therapy remains doubtful in view of the almost constant pathological finding of haemorrhagic infarction, as in our patient.

In pregnancy it seems likely that a coagulopathy is contributory to the development of cerebral venous thrombosis, there being an increase in platelet number and adhesiveness as well as of clotting factors VII, IX, and fibrinogen and also a decrease in fibrinolysis (Gillman et al., 1959; Pechet and Alexander, 1961; Amias, 1970; Gettelfinger and Kokmen, 1977). Another important contributory factor may be the predisposition of the cortical venous system to thrombotic episodes, by virtue of its lack of valves, low pressure, fibrous septae, and transient changes in pressure during pregnancy (Amias, 1970). Embolism of pelvic 
thrombi via the paravertebral venous plexus to the cerebral veins as suggested by Batson (1940) has been proposed in this situation by Kalbag and Woolf (1967).

Whatever the mechanism, the rarity of cerebral venous thrombosis in early pregnancy is remarkable. There are only eight necropsy proven cases described in the literature, occurring during the first trimester of pregnancy (Meinel, 1901; Fishman et al., 1957; Hensell, 1961; Eckerling et al., 1963; Kalbag and Woolf, 1967; Christensen et al., 1969). These show an age range of 20 to 42 years. Only two were known to be multiparous (Eckerling et al., 1963; Kalbag and Woolf, 1967). Extensive cortical venous thrombosis with haemorrhagic infarction was found in all but two cases (Fishman et al., 1957; Christensen et al., 1969). In three cases there was a complication of pregnancy (Meinel, 1901; Fishman et al., 1957; Kalbag and Woolf, 1967). In none of the eight cases was thrombosis of the systemic veins reported. The presence of thrombosis in the pelvic and iliac veins in our patient would support the mechanisms of either a generalised coagulopathy or embolism via the paravertebral venous plexus. The role of the placental separation is unknown but it could have contributed to or caused the generalised coagulopathy, or, in fact, have been the result of a seizure. However, the development of symptoms early in pregnancy, probably as early as two weeks gestation in our patient, must raise the question of a hormonal role in the mechanism of a coagulopathy such as probably occurs with the oestrogen containing contraceptive pill (Inman et al., 1970).

Kalbag and Woolf (1967) found their patient to have thrombosis in an advanced state of organisation and suggested that the process might have commenced in a previous pregnancy.

Antemortem diagnosis in the past has depended on the angiographic appearances of delay or defect in venous filling and persistence of the venous phase. Computerised axial tomography with the appearances of bilateral cerebral venous infarction taken in conjunction with the clinical picture makes possible the diagnosis, intra vitam, of this disorder. However, in the early stages or milder form, cerebral angiography remains desirable.

We wish to thank Dr Simon Currie for his permission to report the case.

\section{References}

Amias, A. G. (1970). Cerebral vascular disease in pregnancy. Journal of Obstetrics of the British Commonwealth, 77, 312-325.

Batson, D. V. (1940). Function of the vertebral veins. Annals of Surgery, 112, 138-149.

Carroll, J. D., Leak, D., and Lee, H. A. (1966). Cerebral thrombophlebitis in pregnancy and the puerperium. Quarterly Journal of Medicine, 35, 347-368.

Christensen, E., Gormsen, J., and Rosenklint, A. (1969). Thrombosis in the superior sagittal sinus during pregnancy. Ugeskrift for Laeger, 131, 869873.

Eckerling, B., Goldman, J. A., and Gans, B. (1963). Intracranial sinus thrombosis: a rare complication of pregnancy ( 3 cases). Obstetrics and Gynaecology, 21, 368-371.

Fishman, R. A., Cowen, D., and Silbermann, M. (1957). Intracranial venous thrombosis during the first trimester of pregnancy. Neurology (Minneapolis), 7, 217-220.

Garcin, R., and Pestel, M. (1955). Thrombo-phlébites cérébrales. XXXe Congrès France de Médecine, Algiers. Pp. 503-533. Association des Medicins de Langue Française: Paris.

Gettelfinger, D. M., and Kokmen, O. (1977). Superior sagittal sinus thrombosis. Archives of Neurology (Chicago), 34, 2-6.

Gillman, T., Naidoo, S. S., and Hathorn, M. (1959). Plasma fibrinogen activity in pregnancy. Lancet, 2, 70-71.

Gowers, W. R. (1888). On thrombosis in the cerebral sinuses and veins. In $A$ Manual Of Diseases of the Nervous System. Vol. 2, p. 416. Churchill: London.

Hensell, V. (1961). Traumatische Hirnsinus-und Venenthrombosen. Acta Neurochirurgica, Supplement 7, 362-366.

Huggenberg, H. R., and Kesselring, F. (1958). Post partum cerebral complications. Gynaecologia, 146, 312-317.

Inman, W. H. W., Vessey, M. P., Westerholm, B., and Engelund, A. (1970). Thromboembolic disease and the steroidal content of oral contraceptives. A report to the Committee on Safety of Drugs. British Medical Journal, 2, 203-209.

Kalbag, R. M., and Woolf, A. L. (1967). Cerebral Venous Thrombosis. Oxford University Press: London.

Meinel, A. (1901). Ueber das Vorkommen des autochthonen Sinus Thrombose bei Chlorose (Inaugural dissertation, Münich). Quoted by Kalbag and Woolf (1967).

Pechet, L., and Alexander, B. (1961). Increased clotting factors in pregnancy. New England Journal of Medicine, 265, 1093-1097. 